\title{
Prevalência de hipertensão arterial sistêmica em idosos no Brasil entre 2006 e 2010
}

\author{
Prevalence of hypertension in elderly in Brazil between 2006 and 2010 \\ Prevalencia de hipertensión arterial sistémica en ancianos en Brasil entre 2006 y 2010 \\ Gisele Soares Mendes. Universidade Católica de Brasília (UCB). Brasília, DF, Brasil. gsmendes.fisio@gmail.com (Autora correspondente) \\ Clayton Franco Moraes. Universidade Católica de Brasília (UCB). Brasília, DF, Brasil. claytonf@ucb.br \\ Lucy Gomes. Universidade Católica de Brasília (UCB). Brasília, DF, Brasil. lucygomes@pos.ucb.br
}

\section{Resumo}

Objetivo: observar a evolução da prevalência de hipertensão arterial sistêmica (HAS) em idosos entre 2006 e 2010 no Brasil. Métodos: estudo descritivo, ecológico, quantitativo, de um período entre 2006 e 2010, com dados coletados do Departamento de Informática do Sistema Único de Saúde (DATASUS) relacionados ao sexo, região e escolaridade na faixa etária de 65 anos ou mais. Resultados: na análise entre regiões brasileiras, não houve diferença significativa nos anos analisados. Na comparação entre os sexos, a prevalência de HAS acima de 65 anos nas mulheres foi maior que nos homens. Com relação ao nível de escolaridade, foi encontrado que a prevalência no ano de 2006 foi significativamente menor do que nos anos de 2008 e 2009 entre pessoas com 9 a 11 anos de educação formal. Entre as regiões, quanto menor o nível de escolaridade, maior a prevalência de hipertensão. Conclusão: a prevalência da HAS acima dos 65 anos não seguiu uma tendência linear, mas manteve-se elevada, com predomínio em idosos do sexo feminino e em idosos com baixa escolaridade, chamando a atenção para a necessidade de ações de prevenção dos fatores de risco e acompanhamento em longo prazo dos idosos hipertensos.

\section{Abstract}

Objective: to observe the evolution of the prevalence of hypertension in the Brazilian elderly population between 2006 and 2010. Methods: descriptive, ecological, quantitative study of the prevalence of hypertension among the Brazilian population aged 65 years or above, with data collected through telephone interviews from 2006 to 2010, obtained from the Information Technology Department of the Brazilian Public Health System (DATASUS), related to sex, region and education. Results: we found no significant difference in the prevalence of hypertension across Brazilian regions in the period analyzed. In women aged 65 years or above, the prevalence of hypertension was higher than in men in that same age group. With regard to education, we found that in people with 9-11 years of schooling the prevalence of hypertension in 2006 was significantly lower than in 2008 and 2009. Across Brazilian regions, the lower the education level the higher the prevalence of hypertension. Conclusion: The prevalence of hypertension in the population aged 65 years or above did not follow a linear trend but remained elevated, predominantly in elderly females and elderly people with low schooling, calling attention to the importance of preventing risk factors and to the long-term follow-up of people with hypertension.

\section{Resumen}

Objetivo: observar la evolución de la prevalencia de la hipertensión arterial (HTA) en los ancianos entre 2006 y 2010 en Brasil. Métodos: estudio descriptivo, ecológico, cuantitativo, de un periodo comprendido entre 2006 a 2010 con datos recogidos del Departamento de Informática del Sistema Único de Salud (DATASUS), relacionada con el sexo, la región y la educación en un grupo de edad de 65 años o más. Resultados: en el análisis entre las regiones de Brasil no hay diferencia significativa en los años analizados. En la comparación entre los sexos, la prevalencia de hipertensión en personas con 65 años o más fue mayor en mujeres que en hombres. En la escolaridad, fue encontrado que, con 9-11 años de estudio, la prevalencia en 2006 fue significativamente menor que en los años 2008 y 2009. Entre las regiones, cuanto menor sea el nivel de escolaridad, mayor es la prevalencia de la hipertensión. Conclusión: la prevalencia de la hipertensión en personas con más de 65 años no siguió una tendencia lineal, sino que se mantuvo elevada, sobre todo en mujeres de edad avanzada y en las personas mayores con baja escolaridad, llamando la atención sobre las acciones preventivas y de vigilancia de factores de riesgo en largo plazo en los hipertensos.

\section{Palavras-chave:}

Idoso

Hipertensão

Vigilância Sanitária

Prevalência

Brasil

\section{Keywords:}

Aged

Hypertension

Health Surveillance

Prevalence

Brazil 


\section{Introdução}

O aumento da população idosa é um fenômeno mundial. ${ }^{1,2}$ A população brasileira atual possui mais de 201 milhôes de pessoas, sendo que 12,6\% têm 60 anos ou mais. Dessas, 55,7\% são mulheres e 44,3\% são homens. ${ }^{3,4}$ Há tendência à inversão no modelo de crescimento populacional, com aumento progressivo dos idosos e redução relativa dos jovens. ${ }^{5}$

O envelhecimento é um processo dinâmico e progressivo que causa diversas alteraçôes no organismo, sejam elas de ordem morfológica, psicológica, funcional ou biológica, levando à diminuiçáo da capacidade funcional e ao desenvolvimento de doenças crônicas não-transmissíveis. ${ }^{6,7}$ Dentre os fatores de risco para o desenvolvimento das doenças crônicas não-transmissíveis, a hipertensão arterial sistêmica (HAS) é o mais prevalente. ${ }^{8-10}$

A HAS é definida como a manutenção de níveis de pressão arterial acima de $140 \mathrm{mmHg}$ na sistólica e $90 \mathrm{mmHg}$ na diastólica. ${ }^{11}$ Está relacionada a fatores intrínsecos, como hereditariedade, sexo, idade e raça; e a fatores extrínsecos, como tabagismo, sedentarismo, obesidade, estresse, dislipidemia e dieta. ${ }^{12}$ Além disso, há aumento do risco de comorbidades, como infarto agudo do miocárdio, acidente vascular encefálico e insuficiência renal crônica. ${ }^{8}$

A HAS é um importante problema de saúde pública, visto que a morbimortalidade e os custos com o seu tratamento são elevados. ${ }^{9}, 13$ Por ser muitas vezes assintomática, há dificuldades para que os indivíduos procurem os serviços de saúde para o diagnóstico e adesão ao tratamento. ${ }^{14}$ Somam-se ainda a falta de estrutura dos sistemas de saúde para atender a essa população e as escassas açóes preventivas para reduzir os fatores de risco. ${ }^{5,15}$

Com o objetivo de realizar o monitoramento da frequência e da distribuição das principais doenças crônicas não transmissíveis e seus fatores de risco, o Ministério da Saúde (MS) realiza desde 2006 um inquérito telefônico nas 26 capitais brasileiras e no Distrito Federal, denominado Vigilância de Fatores de Risco e Proteção para Doenças Crônicas por Inquérito Telefônico (VIGITEL). ${ }^{16}$

Haja vista que a HAS é um problema de saúde pública e ainda necessita de açôes de maior vulto para seu acompanhamento e tratamento, o objetivo do presente estudo foi o de observar a evoluçáo da prevalência de HAS em indivíduos idosos no Brasil, entre os anos de 2006 e 2010.

\section{Métodos}

O presente estudo foi um painel ecológico, descritivo, quantitativo e de série histórica, que abrangeu o período de 2006 a 2010, com dados coletados no Departamento de Informática do Sistema Único de Saúde (DATASUS), por meio do site $<$ http://www.datasus.gov.br/>. A seleção da amostra foi realizada a partir da plataforma Informaçóes de Saúde (TABNET), item "Indicadores de Saúde". Dentro deste, foi selecionado "Indicadores e Dados Básicos (IDB)", que apresenta uma série de indicadores demográficos, socioeconômicos, de riscos, mortalidade e cobertura. Para este trabalho, utilizou-se o indicador de fatores de risco e proteção, no qual se encontra a prevalência de HAS, baseada nas coletas realizadas pela VIGITEL. Os dados foram coletados no mês de abril de 2013.

A partir dessa plataforma, os dados da prevalência de HAS na faixa etária de 65 anos ou mais, para os anos de 2006 a 2010, foram relacionados ao sexo (masculino e feminino), escolaridade ( 0 a 8 anos, 9 a 11 anos, 12 anos ou mais) e regiâo geográfica (Centro-Oeste, Norte, Nordeste, Sudeste e Sul), sendo a unidade de análise as capitais de cada Unidade da Federação quando se compararam as prevalências ao longo dos cinco anos dentro de cada região geográfica, e as regióes geográficas quando se compararam as prevalências ao longo dos anos dentro de cada nível educacional ou sexo. Não houve critério de exclusão.

A análise estatística foi realizada usando-se SAS 9.3. As médias de prevalência de HAS foram comparadas com o uso de modelos de análise de variância (ANOVA) a dois fatores fixos com interação. Comparaçóes entre os anos, dentro de cada região geográfica, sexo e escolaridade, foram realizadas pelo teste $\mathrm{F}$ para efeitos simples. ${ }^{17}$ Quando o teste $\mathrm{F}$ mostrou-se significativo entre os anos, foi empregado o teste de Fisher Least Significant Difference (LSD) para as comparaçóes múltiplas post-hoc. Foi realizada análise de resíduo para verificar se os resíduos dos modelos apresentavam distribuição gaussiana com variância constante, constatando-se que esses pressupostos foram observados em todos os modelos realizados. Os resultados foram apresentados em termos de média \pm desvio padrão. $\mathrm{O}$ valor $\mathrm{p}<0,05$ foi estabelecido como o critério para a significância estatística. 


\section{Resultados}

As prevalências de HAS em idosos nas cinco regióes geográficas brasileiras nos anos de 2006 a 2010 estão na Tabela 1. Nas cinco regiôes, a prevalência média de HAS em ambos os sexos e níveis de escolaridade não diferiu ao longo dos cinco anos. Os valores encontrados nas cinco regiôes estão acima de 50\% em todos os anos analisados, porém não seguem uma tendência linear.

Tabela 1. Prevalência de HAS nos idosos brasileiros, segundo a região geográfica, 2006-2010.

\begin{tabular}{lcccccc}
\hline \multirow{2}{*}{ Região } & \multicolumn{5}{c}{ Anos } & p-valor \\
\cline { 2 - 5 } & 2006 & 2007 & 2008 & 2009 & 2010 & 0 \\
\hline Norte & $58,8 \pm 5,3$ & $56,8 \pm 7,2$ & $57,4 \pm 5,2$ & $61,1 \pm 5,1$ & $61,2 \pm 4,5$ & 0,2416 \\
Nordeste & $58,4 \pm 5,0$ & $59,1 \pm 3,6$ & $59,1 \pm 5,5$ & $60,9 \pm 4,7$ & $61,9 \pm 2,9$ & 0,4734 \\
Centro Oeste & $59,2 \pm 4,0$ & $57,4 \pm 2,8$ & $61,5 \pm 3,6$ & $65,1 \pm 3,3$ & $57,9 \pm 7,0$ & 0,1177 \\
Sudeste & $56,9 \pm 1,8$ & $57,3 \pm 4,2$ & $61,1 \pm 2,2$ & $63,7 \pm 6,1$ & $63,1 \pm 4,8$ & 0,1152 \\
Sul & $55,0 \pm 2,8$ & $58,1 \pm 2,6$ & $62,5 \pm 2,9$ & $59,4 \pm 3,2$ & $59,1 \pm 1,4$ & 0,4052 \\
\hline
\end{tabular}

Fonte: dos autores, elaborado com dados coletados no DATASUS.

Analisando-se os sexos, não surgiu diferença significativa na prevalência média de HAS em idosos do sexo feminino nos anos estudados. No sexo masculino, houve diferença significativa em 2006 e 2007 com relação ao ano de 2009. Em todos os anos, a prevalência de HAS nas mulheres foi maior que nos homens (Figura 1).

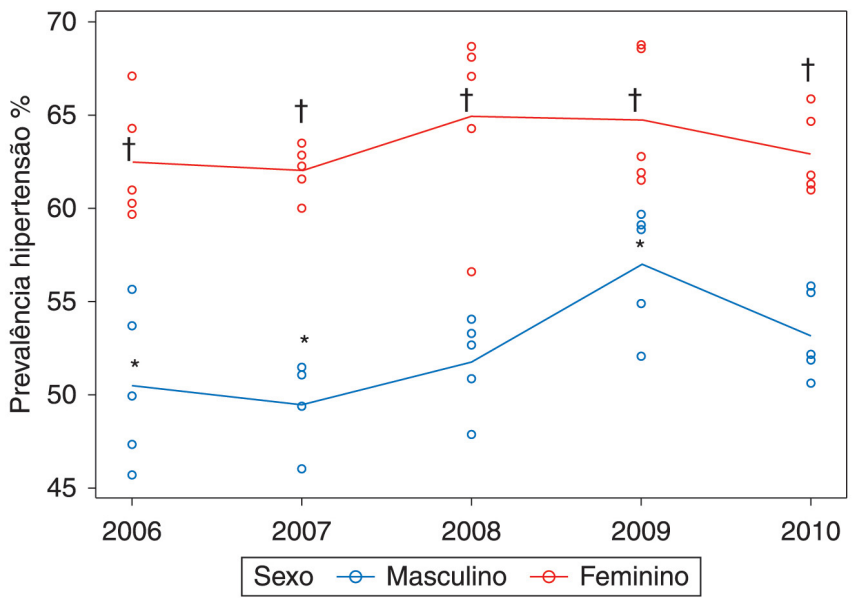

Figura 1. HAS no Brasil, segundo os sexos, 2006-2010.

*Diferença significativa em 2006 e 2007 com relação a 2009.

†Diferença significativa entre os sexos. Fonte: dos autores, elaborado com dados coletados no DATASUS.

Com relação ao nível de escolaridade, verificou-se que, para 0 a 8 anos e $\geq 12$ anos de educação formal, a prevalência média de HAS não diferiu ao longo dos cinco anos. Porém, de 9 a 11 anos de educação formal, a prevalência em 2006 foi significativamente menor do que nos anos de 2008 e 2009. Náo houve diferença significativa nos outros anos (Tabela 2).

Tabela 2. HAS nos indivíduos das diferentes regiões geográficas brasileiras, segundo o grau de escolaridade.

\begin{tabular}{|c|c|c|c|c|c|c|}
\hline \multirow{2}{*}{ Escolaridade } & \multicolumn{5}{|c|}{ Anos } & \multirow{2}{*}{ p-valor } \\
\hline & 2006 & 2007 & 2008 & 2009 & 2010 & \\
\hline 0 a 8 anos & $59,58 \pm 0,43$ & $58,80 \pm 2,36$ & $61,36 \pm 4,25$ & $63,40 \pm 3,97$ & $60,66 \pm 2,93$ & 0,5784 \\
\hline 9 a 11 anos & $50,28 \pm 2,97^{*}$ & $52,90 \pm 4,80$ & $58,58 \pm 5,34^{*}$ & $57,20 \pm 2,76^{*}$ & $54,40 \pm 1,37$ & 0,0485 \\
\hline$\geq 12$ anos & $49,72 \pm 6,19$ & $48,06 \pm 6,02$ & $46,42 \pm 9,49$ & $53,42 \pm 5,65$ & $51,98 \pm 3,24$ & 0,1295 \\
\hline
\end{tabular}

*Prevalência significativamente superior com relação à 2006.

Fonte: dos autores, elaborado com dados coletados no DATASUS. 
Nas regióes Centro-Oeste e Norte, a HAS em indivíduos com 0 a 8 anos de educação formal não diferiu significativamente daqueles com 9 a 11 anos de educação formal. Nessas regiôes, a HAS nos sujeitos com 0 a 8 e com 9 a 12 anos de escolaridade foi significativamente maior do que nos com 12 ou mais anos.

Nas regiōes Nordeste e Sudeste, a média de HAS nos indivíduos com 0 a 8 anos de educação formal foi significativamente maior que as médias naqueles com 9 a 11 anos e 12 ou mais anos de escolaridade. Na regiáo Sul, a prevalência média de HAS nos indivíduos com 0 a 8 anos de escolaridade foi significativamente maior que a prevalência média naqueles com 9 a 11 anos, a qual, por sua vez, foi significativamente maior que a prevalência média nos sujeitos com 12 ou mais anos de escolaridade (Figura 2).

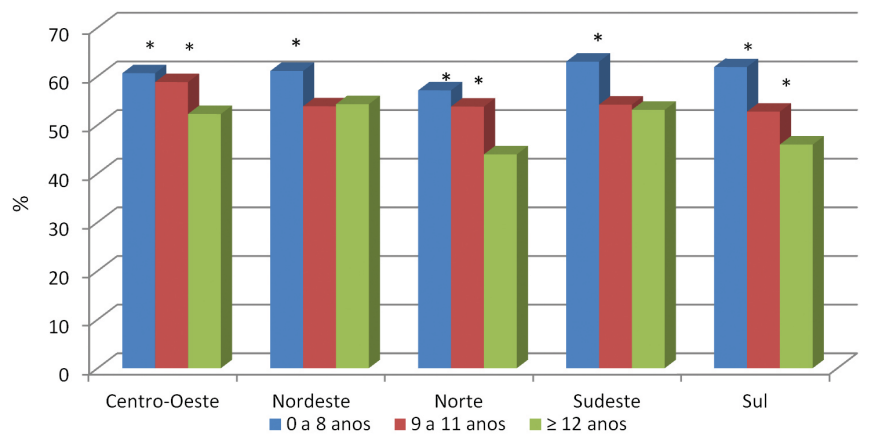

Figura 2. Prevalência de HAS em idosos de acordo com grau de escolaridade e região geográfica entre 2006 e 2010. *Prevalência significativamente maior com relação aos outros níveis de escolaridade dentro da região.

Fonte: dos autores, elaborado com dados coletados no DATASUS.

\section{Discussão}

Em todo o mundo, observa-se alta prevalência de HAS. Estudos realizados na Europa, nos Estados Unidos e no Canadá mostraram números elevados de HAS principalmente na população idosa - entre $30 \%$ e $35 \%{ }^{18-20}$ No presente estudo, a prevalência de HAS foi acima de 55\% da populaçáo idosa em todas as regióes geográficas, valor superior ao encontrado nos países de alta renda. Já em países como a Nigéria e a China, a prevalência é de 66,7\% e 56,5\%, respectivamente, demonstrando que países em desenvolvimento apresentam prevalências de HAS bem mais elevadas do que as dos países de alta renda. ${ }^{21,22}$

No presente estudo, a prevalência de HAS em idosos do sexo feminino foi significativamente maior que no sexo masculino em todos os anos avaliados. Diversos estudos demonstram que as mulheres procuram mais pelos serviços de saúde, aumentando suas chances de terem o diagnóstico de HAS, ${ }^{8,11,13,23}$ além de afirmarem que mulheres, por terem maior sobrevida que homens, ficam mais propensas a sofrerem doenças crônicas. Outros autores ${ }^{24}$ justificaram essa maior prevalência em mulheres devido ao fato de esse grupo apresentar aumento dos fatores de risco com a queda na produçấo de hormônios esteroides, levando ao aumento do tônus vascular das artérias periféricas e provocando HAS em mulheres na pós-menopausa.

Índices mundiais indicam que a diferença na prevalência de HAS entre os sexos é pequena devido à maior prevalência em homens mais jovens e em mulheres mais idosas. ${ }^{11}$ Porém, ao avaliarmos a mesma faixa etária, o sexo feminino apresentou valores maiores. Os homens muitas vezes descobrem que são hipertensos somente após sofrerem evento clínico grave, como infarto ou acidente vascular encefálico. ${ }^{14}$ Isso demonstra a necessidade de investimentos em açóes públicas de saúde para informação, prevenção, diagnóstico e tratamento da população, principalmente de homens idosos, visando com isso a reduzir os agravos advindos da HAS.

O nível de escolaridade é um dos mais importantes indicadores das condiçóes de saúde da população e está intimamente relacionado à idade no Brasil, visto que muitos idosos deste século náo tiveram oportunidades de estudo na juventude. ${ }^{25}$ Este estudo demonstrou que, quanto menor o tempo de educaçáo formal, maior a prevalência de HAS, sendo este achado observado em todas as regiôes geográficas e anos analisados. Esse fato evidencia a desigualdade em saúde, pois indivíduos com menor instrução têm acesso precário ao sistema de saúde, recebem menos informaçóes e não compreendem a importância do problema e da adesão ao tratamento da HAS, ${ }^{15,26}$ demonstrando, mais uma vez, a necessidade de açóes voltadas para a prevenção desse fator de risco para doenças cardiovasculares. 
A partir do SUS, foi criada a Estratégia Saúde da Família (ESF) como forma de garantir os princípios da Atenção Primária à Saúde (APS), tomando medidas de intervenção comunitária, com a finalidade de diagnosticar e acompanhar o indivíduo de forma mais próxima. Apesar de ainda não ter cobertura plena no atendimento primário, a ESF pode ser um importante instrumento para auxiliar na orientação e no acompanhamento do tratamento farmacológico e não farmacológico, bem como das mudanças no estilo de vida e da adoçáo de hábitos saudáveis a longo prazo, em todos os grupos sociais, principalmente aqueles com menores possibilidades de escolha, melhorando o prognóstico e a qualidade de vida de pessoas com hipertensão. ${ }^{27,28}$

No Brasil, a APS é monitorada por meio do Sistema de Informação da Atenção Básica (SIAB), no qual podem ser encontradas informaçóes sobre os casos de HAS. Porém, esse sistema não dispóe do número de casos de HAS por idade, o que dificulta a determinação da faixa etária de risco, interferindo na definição de açóes preventivas voltadas para a mesma. Seria interessante que a utilização do prontuário eletrônico permitisse uma análise detalhada do acompanhamento dos casos, já que o preenchimento dos dados pode ser feito de forma mais prática.

A utilização de dados secundários foi uma das limitaçóes deste estudo, podendo haver viés de informação. Além disso, não foi realizada uma análise multivariada dos dados, limitando a análise das variáveis. A principal limitação das informaçôes coletadas pela VIGITEL é que esse tipo de coleta exclui os indivíduos que não possuem telefone fixo, podendo comprometer significativamente os resultados. Além disso, existe menor controle do ambiente no inquérito por telefone, como interferência de familiares, além da incerteza de se é realmente o amostrado que está respondendo ao questionário. ${ }^{29}$ Por outro lado, existe a ponderação pós-estratificação, que ajusta a distribuição da amostragem por telefone à composição da população total. Assim, ao se utilizar o peso pós-estratificação, o processo de não-resposta torna-se ignorável e não interfere nos resultados. ${ }^{30,31}$

Estudo que analisou a validade externa dos inquéritos telefônicos realizados pela VIGITEL comparados ao inquérito domiciliar face a face em Belo Horizonte concluiu que os resultados encontrados em ambas metodologias foram semelhantes, recomendando a vigilância epidemiológica de doenças crônicas náo transmissíveis por inquérito telefônico, visto que a mesma oferece uma estimativa da população total, com menor investimento financeiro e de tempo do que em investigação domiciliar. ${ }^{16}$

\section{Conclusão}

Observando o comportamento da HAS em idosos no Brasil em período de cinco anos, demonstrou-se que sua prevalência não seguiu uma tendência linear, mantendo-se elevada em todos os anos analisados, com predomínio em idosos do sexo feminino e em idosos com baixa escolaridade. Esses dados chamam a atenção para açôes de prevenção a longo prazo junto dos idosos com HAS, como orientaçôes sobre hábitos de vida (diminuição da ingestão de sal, prática de atividade física, uso correto dos medicamentos), além de um registro e acompanhamento minuncioso dos casos por meio de prontuário eletrônico que permitiria relacionar a HAS com comorbidades cardiovasculares, visto que esse é o maior fator de risco para tais doenças.

\section{Referências}

1. Fernández-Ballesteros R, Robine JM, Walker A, Kalache A. Active Aging: A Global Goal. Curr Gerontol Geriatr Res. 2013;2013:1-4. http://dx.doi.org/10.1155/2013/298012.

2. Tavares DMS, Dias FA. Capacidade funcional, morbidades e qualidade de vida de idosos. Texto Contexto Enferm. 2012;21(1):112-20. http://dx.doi.org/10.1590/S0104-07072012000100013.

3. Instituto Brasileiro de Geografia e Estatística (IBGE). Estimativas da população residente no Brasil e unidades da Federação com data de referência em $1^{\circ}$ de julho de 2013. Rio de Janeiro: IBGE; 2013.

4. Instituto Brasileiro de Geografia e Estatística (IBGE). Síntese de indicadores sociais: uma análise das condições da vida da população brasileira 2013. Rio de Janeiro: IBGE; 2013.

5. Brito CJ, Volp ACP, Nóbrega OT, Silva Júnior FL, Mendes EL, Roas AFCM, et al. Exercício físico como fator de prevenção aos processos inflamatórios decorrentes do envelhecimento. Motriz. 2011;17(3):544-55. http://dx.doi.org/10.1590/S1980-65742011000300017.

6. Montagner S, Costa A. Bases biomoleculares do fotoenvelhecimento. An Bras Dermatol. 2009;84(3):263-69. http://dx.doi.org/10.1590/S0365-05962009000300008.

7. Ferreira OGL, Maciel SC, Costa SMG, Silva AO, Moreira MASP. Envelhecimento ativo e sua relação com a independência funcional. Texto Contexto Enferm. 2012;21(3):513-18. http://dx.doi.org/10.1590/S0104-07072012000300004. 
8. Boing AC, Boing AF. Hipertensão arterial sistêmica: o que nos dizem os sistemas brasileiros de cadastramentos e informações em saúde. Rev Bras Hipertens. 2007 [acesso em 2013 Jul 10];14(2):84-8. Disponível em: http://departamentos.cardiol.br/dha/revista/14-2/06-hipertensao.pdf.

9. Gontijo MF, Ribeiro AQ, Kein CH, Rozenfeld S, Acurcio FA. Uso de anti-hipertensivos e antidiabéticos por idosos: inquérito em Belo Horizonte, Minas Gerais, Brasil. Cad Saúde Pública. 2012; 28(7): 1337-46. http://dx.doi.org/10.1590/S0102-311X2012000700012.

10. Paniz VMV, Fassa AG, Facchini LA, Piccini RX, Tomasi E, Thumé E, et al. Free access to hypertension and diabetes medicines among the elderly: a reality yet to be constructed. Cad Saúde Pública. 2010;26(6):1163-74. http://dx.doi.org/10.1590/S0102-311X2010000600010.

11. Cesarino CB, Cipullo JP, Martin JFV, Ciorlia LA, Godoy MRP, Cordeiro JA, et al. Prevalência e fatores sociodemográficos em hipertensos de São José do Rio Preto - SP. Arq Bras Cardiol. 2008;91(1):31-5. http://dx.doi.org/10.1590/S0066-782X2008001300005.

12. Giroto E, Andrade SM, Cabrera MAS, Ridão EG. Prevalência de fatores de risco para doenças cardiovasculares em hipertensos cadastrados em unidade de saúde da família. Acta Sci, Health Sci. 2009;31(1):77-82. http://dx.doi.org/10.4025/actascihealthsci.v31i1.4492.

13. Borim FSA, Guariento ME, Almeida EA. Perfil de adultos e idosos hipertensos em unidade básica de saúde. Rev Soc Bras Clín Méd. 2011 [acesso em 2013 Jul 10];9(2):107-11. http://files.bvs.br/upload/S/1679-1010/2011/v9n2/a1832.pdf.

14. Zattar LC. Prevalência e fatores associados à pressão arterial elevada, seu conhecimento e tratamento em idosos no sul do Brasil. Cad Saúde Pública. 2013;29(3):507-21. http://dx.doi.org/10.1590/S0102-311X2013000300009.

15. Gus I, Harzheim E, Zaslavsky C, Medina C, Gus M. Prevalência, reconhecimento e controle da hipertensão arterial sistêmica no estado do Rio Grande do Sul. Arq Bras Cardiol. 2004;83(5):424-28. http://dx.doi.org/10.1590/S0066-782X2004001700009.

16. Ferreira AD, César CC, Malta DC, Andrade ACS, Ramos CGC, Proletti FA, et al. Validade de estimativas obtidas por inquérito telefônico: comparação entre VIGITEL 2008 e Inquérito Saúde em Beagá. Rev Bras Epidemiol. 2011;14(Suppl 1):16-30. http://dx.doi.org/10.1590/S1415-790X2011000500003.

17. Winer BJ, Brown DR, Michels KM. Statistical Principles in Experimental Design. 2a ed. New York: McGraw-Hill; 1971.

18. Allen M, Kelly K, Fleming I. Hypertension in elderly patients recommended systolic targets are not evidence based. Can Fam Physician. 2013 [acesso em 2013 Jul 10];59:19-21. Disponível em: http://www.cfp.ca/content/59/1/19.full.

19. Chrysant SG. Treating blood pressure to prevent strokes: the age factor. World J Cardiol. 2013;5(3):22-7. http://dx.doi.org/10.4330/wjc.v5.i3.22.

20. Olives C, Myerson R, Mokdad AH, Murray CJL, Lim SS. Prevalence, awareness, treatment, and control of hypertension in United States countries, 2001-2009. PloS ONE. 2013;8(4):1-8. http://dx.doi.org/10.1371/journal.pone.0060308.

21. Asekun-Olarinmoye EO, Akinwuse PO, Adebimpe WO, Isawumi MA, Hassan MB, Olowe OA, et al. Prevalence of hypertension in the rural adult population of Osun State, southwestern Nigeria. Int J Gen Med. 2013;6:317-22. http://dx.doi.org/10.2147/IJGM.S42905.

22. Gao Y, Chen G, Tian H, Lin L, Lu J, Weng J, et al. Prevalence of hypertension in China: a cross-sectional study. PLoS ONE. 2013;8(6):1-8. http://dx.doi.org/10.1371/journal.pone.0065938.

23. Diniz MA, Tavares DMS, Rodrigues LR. Características sócio-demográficas e de saúde entre idosos com hipertensão arterial. Ciênc Cuid Saúde. 2009;8(4):607-14. http://dx.doi.org/10.4025/cienccuidsaude.v8i4.9689.

24. Oca-Rodríguez A, Naranjo-Herrera Y, Medina-González G, Hernández-Martinéz B, Jorge-Molina M. Características clínico-epidemiológicas de la hipertensión arterial con relación a variables modificables y no modificables. Rev Soc Peru Med Interna. 2012;25(2):70-3.

25. Barros MBA, Francisco PMSB, Lima MG, César CLG. Social inequalities in health among the elderly. Cad Saúde Pública. 2011;27(Suppl 2):198208. http://dx.doi.org/10.1590/S0102-311X2011001400008.

26. Zavatini MA, Obreli-Neto PR, Cuman RKN. Estratégia saúde da família no tratamento de doenças crônico-degenerativas: avanços e desafios. Rev Gaúcha Enferm. 2010 [acesso em 2013 Jul 10];31(4):647-54. Disponível em: http://seer.ufrgs.br/RevistaGauchadeEnfermagem/article/view/13275.

27. Cotta RMM, Batista KCS, Reis RS, Souza GA, Dias G, Castro FAF, et al. Perfil sociossanitário e estilo de vida de hipertensos e/ou diabéticos, usuários do Programa de Saúde da Família no município de Teixeiras, MG. Ciênc Saúde Coletiva. 2009;14(4):1251-60. http://dx.doi.org/10.1590/S1413-81232009000400031.

28. Trindade TG. Associação entre extensão dos atributos de Atenção Primária e qualidade do manejo da hipertensão arterial em adultos adscritos à rede de Atenção Primária à Saúde de Porto Alegre [Dissertação]. Porto Alegre: Universidade Federal do Rio Grande do Sul; 2007.

29. Waldman EA, Novaes HMD, Albuquerque MFM, Latorre MRDO, Ribeiro MCSA, Vasconcellos M, et al. Inquéritos populacionais: aspectos metodológicos, operacionais e éticos. Rev Bras Epidemiol. 2008;11(Suppl 1):168-79. http://dx.doi.org/10.1590/S1415-790X2008000500018.

30. Ministério da Saúde (BR), Secretaria de Vigilância em Saúde, Agência Nacional de Saúde Suplementar. VIGITEL Brasil 2008: Vigilância de fatores de risco e proteção para doenças crônicas por inquérito telefônico. Rio de Janeiro: MS; 2009.

31. Bernal R, Silva NN. Cobertura de linhas telefônicas residenciais e vícios potenciais em estudos epidemiológicos. Rev Saúde Pública. 2009;43(3):421-26. http://dx.doi.org/10.1590/S0034-89102009005000024. 